\title{
ROSAT OBSERVATIONS OF BRIGHT BL LACERTAE OBJECTS
}

\author{
G. LAMER, H. BRUNNER, R. STAUBERT \\ Astronomisches Institut der Universität Tübingen, Germany
}

\begin{abstract}
We have compiled a sample of $23 \mathrm{X}$-ray and radio selected BL Lacertae objects which have been observed with the Position Sensitive Proportional Counter (PSPC) on board of the ROSAT Satellite. The sample consists of three parts:

In Table I results from 4 objects observed for their known rapid X-ray variability are presented. 5 objects are the BL Lac subset of a complete sample of flat spectrum radio sources with 5 GHz flux densities $>1 \mathrm{Jy}$. Detailed results from this sample will be published in Brunner et al. 1993. The data of the 14 remainig objects were collected from the ROSAT data archive to supplement the sample. The whole sample contains $7 \mathrm{X}$-ray selected objects $\left(\mathrm{XBLs}, \alpha_{O X}<1.2\right)$ and 16 radio selected objects (RBLs).

The X-ray spectra of the sources are well described by single power laws with galactic absorption. The $\mathrm{X}$-ray energy indices $\alpha_{X}$ are widely dispersed around a mean of 1.34 . Significant $\mathrm{X}$-ray flux variability and correlated spectral variability was detected on timescales down to hours. The object H 1218+304 was found to be rapidly variable within each of three observations. Its spectral hardness is correlated with the flux level (see Table I).

We calculated the intrinsic distributions of the spectral indices $\alpha_{X}$ for the XBL and RBL samples and of the differences between ROSAT and EXOSAT ME spectral indices $\alpha_{P S P C}-\alpha_{M E}$ (only XBL sample) using a maximum likelihood fit. There is no significant difference in the mean spectral indices between the $X$-ray and radio selected subsamples. The mean values $\left\langle\alpha_{X}\right\rangle$ are 1.34 for XBLs and 1.33 for RBLs. The spectra of the X-ray selected objects slightly steepen at higher $\mathrm{X}$-ray energies $\left(\left\langle\alpha_{P S P C}-\alpha_{M E}\right\rangle=-0.11\right)$. This supports the view that the $\mathrm{X}$-ray emission of $\mathrm{XBL}$ is supplied by synchrotron radiation. The steepening of the $\mathrm{X}$-ray spectrum is then due to a cutoff in the energy distribution of the electrons.
\end{abstract}

TABLE I

\begin{tabular}{|c|c|c|c|c|c|c|}
\hline Object & date & $\begin{array}{l}N_{H} \\
\text { gal. }^{1}\end{array}$ & $\underset{2}{N_{H}}$ & $\begin{array}{l}F_{1 k e V} \\
{[\mu J y]}\end{array}$ & $\begin{array}{c}\alpha \\
0.1-2.4 \mathrm{keV}\end{array}$ & $\begin{array}{c}\text { variab. } \\
\text { ampl. }(\Delta t)\end{array}$ \\
\hline $0548-322$ & $92 / 3 / 6$ & 2.94 & $2.76 \pm .14$ & $9.59 \pm .12$ & $0.95 \pm .05$ & - \\
\hline $1218+304$ & $91 / 6 / 15-16$ & & $2.61 \pm .09$ & $12.72 \pm .12$ & $1.19 \pm .03$ & $15 \%(1 \mathrm{~d})$ \\
\hline $1218+304$ & $92 / 6 / 18-22$ & 1.78 & $2.51 \pm .10$ & $10.05 \pm .11$ & $1.22 \pm .03$ & $50 \%(6$ mon $)$ \\
\hline $1218+304$ & $92 / 12 / 8-10$ & & $2.85 \pm .25$ & $5.11 \pm .14$ & $1.47 \pm .07$ & \\
\hline $3 C 371$ & $92 / 4 / 9$ & 4.61 & $3.43 \pm .81$ & $0.32 \pm .02$ & $0.87 \pm .25$ & - \\
\hline 2005-489 & $92 / 4 / 27-29$ & 5.44 & $4.09 \pm .17$ & $5.19 \pm .07$ & $1.92 \pm .04$ & $50 \%$ (6 mon $)$ \\
\hline 2005-489 & $92 / 10 / 28-11 / 1$ & 5.44 & $3.58 \pm .21$ & $2.81 \pm .06$ & $1.91 \pm .05$ & - \\
\hline
\end{tabular}

\section{References}

Brunner, H., Lamer, G., Worrall, D. M., et al., 1993, in preparation

Elvis, M., Lockman, F. J., and Wilkes, B. J., 1989, $A J, 97,777$

Stark, A. A., Gammie, C. F., Wilson, R. W., et al., 1992, ApJS, 79, 77

$$
377
$$

T. J.-L. Courvoisier and A. Blecha: Multi-Wavelength Continuum Emission of AGN, 377.

(C) 1994 IAU. Printed in the Netherlands. 